\title{
Local stress analysis in an SMA during stress-induced martensitic transformation by Kossel microdiffraction
}

\author{
Denis Bouscaud ${ }^{1, a^{*}}$, Sophie Berveiller ${ }^{1, b}$, Raphaël Pesci ${ }^{1, c}$, Etienne Patoor ${ }^{1, d}$ \\ and Adam Morawiec ${ }^{2, e}$ \\ ${ }^{1}$ LEM3 UMR CNRS 7239, ENSAM-Arts et Métiers ParisTech, 4 rue Augustin Fresnel, \\ F-57078 Metz, France \\ ${ }^{2}$ Institute of Metallurgy and Materials Science, Polish Academy of Sciences, Reymonta 25, \\ PL-30-059 Kraków, Poland \\ adenis.bouscaud@ensam.eu, bsophie.berveiller@ensam.eu, 'raphael.pesci@ensam.eu, \\ detienne.patoor@ensam.eu, ${ }^{e}$ nmmorawi@cyf-kr.edu.pl
}

Keywords: scanning electron microscopy; Kossel microdiffraction; strain; in situ tensile straining; martensitic transformation.

\begin{abstract}
.
The Kossel microdiffraction in a scanning electron microscope allows for local stress determination. This technique has been applied to monitor stress evolution within grains of austenite in the course of martensitic transformation in a shape memory alloy. Kossel diffraction patterns were recorded during in situ tensile straining of $\mathrm{Cu}-\mathrm{Al}-\mathrm{Be}$ alloy. These innovative measurements show large stress heterogeneities between grains, with the stress ratio exceeding two. As martensite variants are stress-induced, shear stress components appear in individual grains of austenite.
\end{abstract}

\section{Introduction}

The distinctive properties of shape memory alloys (SMA) are based on a reversible martensitic transformation induced by an external stress or a temperature variation. This transformation is accompanied by a large reversible strain that can reach $5 \%$ in a polycrystal [1]. This stress-induced phenomenon is the well-known effect of superelasticity in SMA.

In order to explain the mechanism causing the motion of internal interfaces between austenite and martensite, many experimental works have been done on single crystals during uniaxial loading and/or temperature changes. The influence of crystallographic orientation on the occurrence of martensitic transformation has been clearly demonstrated [1]. These results, however, cannot be easily extended to martensitic transformation in polycrystalline materials, because the strain incompatibilities occurring at grain boundaries and the stress transfer between transforming grains strongly influence the transformation kinetics [2]. As a consequence, the macroscopic behavior of polycrystals differs from that of single crystals. Micromechanical transition models predict the occurrence of large strain incompatibilities between crystallites [3], but few experimental data are available at this length scale. Synchrotron diffraction experiments on a $\mathrm{Cu}-\mathrm{Al}-\mathrm{Be}$ polycrystal with coarse grains [4] showed that individual grains rotate during uniaxial loading and split into subdomains of different orientations during the martensitic transformation. Moreover, a broadening of austenite X-ray diffraction peaks was observed during the transformation for $\mathrm{Cu}-\mathrm{Al}-\mathrm{Be}[5]$ and $\mathrm{Cu}-$ Al-Zn-Mn [6] polycrystals. It was ascribed to the development of strain incompatibilities during the transformation [5]. The reverse process was observed upon unloading, when martensite transformed back to austenite [6]. Such preliminary results highlight the need for developing experimental methods coupling the characterization of the material behavior with observations of micromechanisms occurring during the martensitic transformation and in situ stress determination. 
In this context, Kossel microdiffraction is a suitable technique to study the evolution of the austenite stress state in individual grains. Performed inside the chamber of a scanning electron microscope, it allows for observing the microstructure (formation of martensite variants) and for determining stress state at the same time. This technique has already been applied to study pseudoelasticty in a Cu-Al-Ni SMA single crystal [7]. In that case, only one strain measurement was carried out on a thin disc deformed from a flat shape to a dome-shaped shell, without knowing the applied loading. In our study, the stress evolution during stress-induced martensitic transformation was measured in 20 grains of a polycrystal for four known external loads.

\section{Recording and analysis of experimental Kossel patterns}

The Kossel patterns are produced in a scanning electron microscope and captured by an X-ray sensitive 2D detector. An energetic electron beam focused on a specimen causes excitation of atoms and emission of X-rays within a volume of few $\mu \mathrm{m}^{3}$. The X-ray radiation is diffracted by the crystallographic planes, and - according to the geometric description of X-ray diffraction - this leads to Kossel cones generated at the point where the electron beam hits the specimen. A cone axis coincides with the normal to the diffracting plane, and the semi-apex angle of the cone equals $90^{\circ}$ minus the Bragg angle. A given Kossel pattern consists of a set of conics - intersections of the Kossel cones with a detector. The location of Kossel lines is sensitive to the lattice strain; the more curved the line, the better the strain sensitivity. An example of experimental pattern is shown in Fig. 1(a). About twenty conics are clearly visible on this pattern. Some of them are strongly curved, and even complete ellipses are observed.
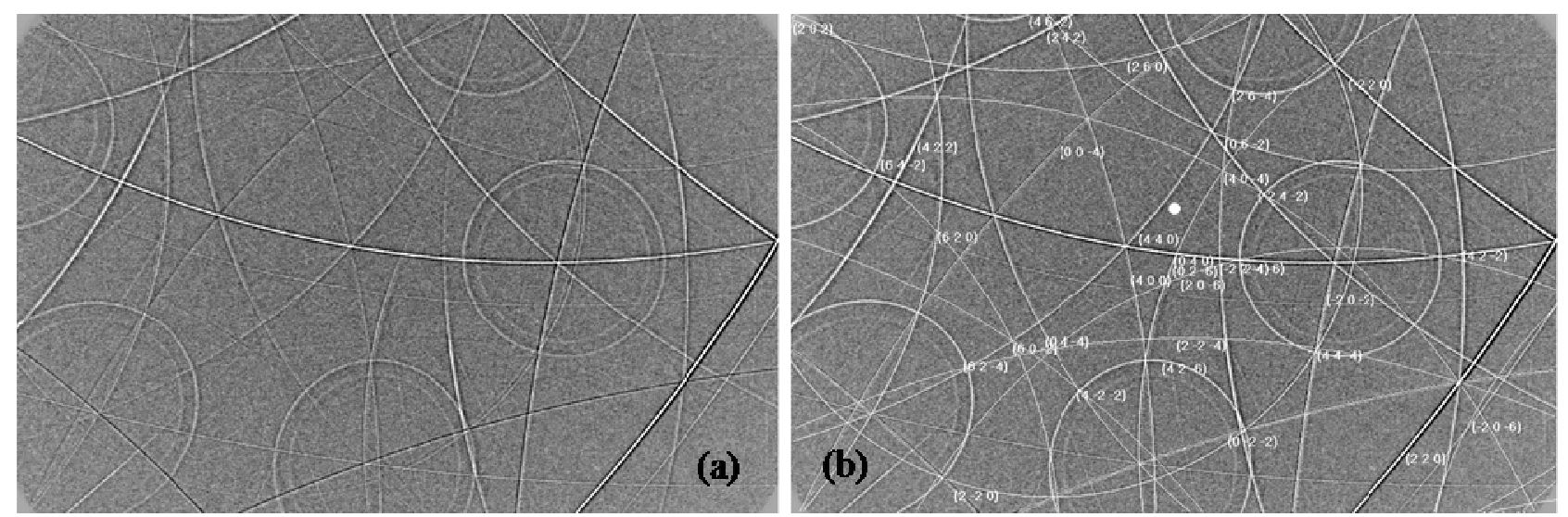

Fig. 1: (a) Experimental Kossel pattern obtained from a Cu-Al-Be crystallite. (b) The corresponding simulated pattern after the strain calculation.

Digitally recorded patterns are directly analyzed using a dedicated software package KSLStrain [8]. The input data are the microscope settings (the sample tilt angle $\left(40^{\circ}\right)$, the detector pixel size $(36 \mu \mathrm{m})$, approximate location of the pattern center $(0,0)$ and approximate sample-to-detector distance $(32,5 \mathrm{~mm}))$, the material properties (the X-ray wavelength $(0.15406 \mathrm{~nm})$ and the crystal structure (see next section)) and the locations of Kossel lines on the pattern. Precise determination of the locations of Kossel lines is needed for the strain analysis. It is carried out by manual positioning of line markers (with the resolution of one tenth of the pixel size) using an intensity profile in the direction perpendicular to the Kossel line. With the input parameters and locations of the markers, the Kossel lines are automatically indexed (Fig. 1b), and the full strain tensor $\varepsilon$ is calculated via the refinement of lattice parameters. The calculation is based on an optimization procedure minimizing deviations between marked locations on experimental lines and lines in kinematically simulated patterns [9]. The strain resolution is affected by the finite thickness and composite profiles of Kossel lines, and it depends on the quality of diffraction patterns, the number of lines used in strain computation and the curvature of these lines. In the case of high quality SMA 
patterns, the resolution of $2 \times 10^{-4}$ is reached for all strain tensor components. The full stress tensor $\boldsymbol{\sigma}$ is calculated using Hooke's law $\boldsymbol{\sigma}=\boldsymbol{C}: \boldsymbol{\varepsilon}$, where $\boldsymbol{C}$ is the elastic stiffness tensor of the crystal. In this study, an additional program was used for deducing the full strain tensor automatically by comparing the patterns obtained during stress-induced martensitic transformation to the one recorded without applying an external stress.

\section{Material and experimental procedure}

The chemical composition (in weight \%) of the investigated alloy was: $\mathrm{Cu}$ - base, $\mathrm{Al}-11.5 \%$ and $\mathrm{Be}-0.5 \%$. The key characteristic property of all SMA is the occurrence of a martensitic phase transformation. The austenitic and the martensitic phases have DO3-type cubic and orthorhombic structures, respectively. The specimen was heat-treated to be $100 \%$ austenitic at room temperature. A dog-bone sample was machined and the surface was then polished (mechanically and electrolytically). The grain size was about few $\mathrm{mm}^{2}$, and the sample thickness was $1.4 \mathrm{~mm}$. In Kossel patterns only the lines diffracted by austenite were considered. One needs to note that austenite is highly anisotropic; the stiffness tensor components used in the stress calculation were $\mathrm{C}_{11}=141.6, \mathrm{C}_{12}=127.4$ and $\mathrm{C}_{44}=94.2 \mathrm{GPa}[10]$.

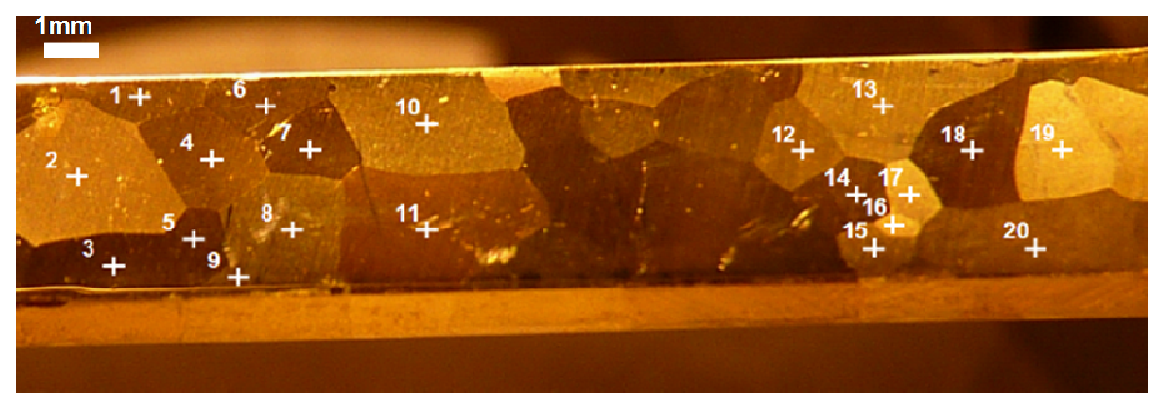

Fig. 2: Sample used for in situ stress analysis, and locations of the 20 measurements.

The scanning microscope used was a Jeol 5800 with thermionic emission tungsten filament operating at the voltage of $30 \mathrm{kV}$. Kossel patterns were recorded using an $11 \mathrm{Mpx}$ high resolution 12bit Peltier-cooled CCD camera ('VHR-11', Photonic Science Ltd.). Strains in the austenitic phase in 20 crystallites (with a single measurement located near grain centre) were determined during uniaxial straining (Fig. 2). Kossel patterns were recorded at the initial state (zero applied stress), for three uniaxial applied stress values and after the complete unloading of the specimen. With this approach, the internal stresses obtained by the Kossel technique can be directly compared with the known values of the applied stress. Tensile tests were carried out in the SEM using a $5 \mathrm{kN}$ tensile/compression module (MicroMecha); we did not use a strain gauge on the specimen.

\section{Macroscopic behavior}

The stress-strain curve of the specimen is plotted in Fig. 3. For lower stresses, as the austenite deforms, the behavior is linear elastic. Microstructural observations showed that the first grains started to transform at the applied stress of $60 \mathrm{MPa}$. Starting at $85 \mathrm{MPa}$, the macroscopic stress remains almost constant despite increasing macroscopic strain. This plateau stress corresponds to the development of martensitic transformation: the martensite appears in new grains and its fraction increases in already transforming grains. The martensite either propagates through the whole grain, or it stays located only in a part of the grain in just one or a few variants (Fig. 4). At the last loading point, some grains still remain untransformed. During unloading, a partial reversible transformation occurs; retained martensite leading to a residual macroscopic strain was observed. 


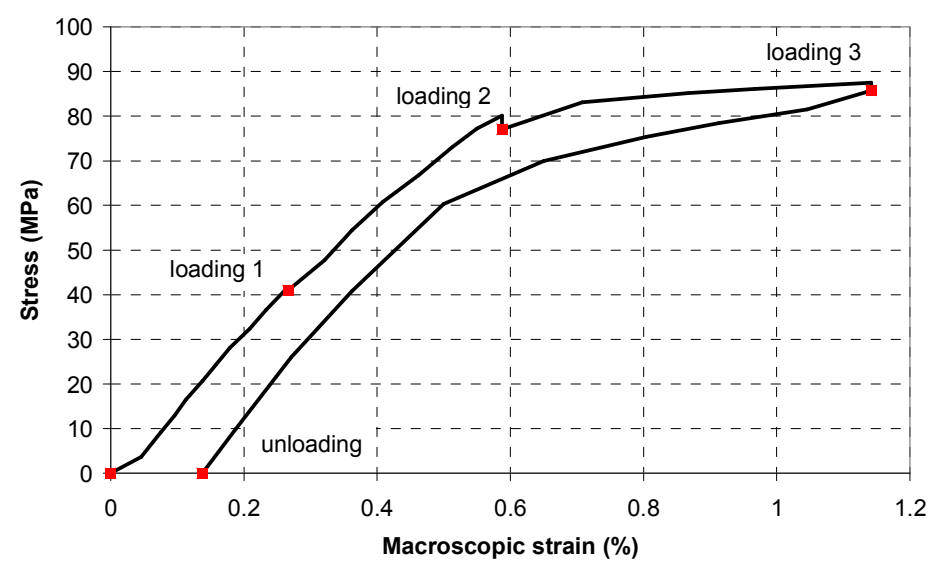

Fig. 3: Strain-stress curve for Cu-Al-Be. Squares mark the strains at which Kossel patterns were recorded.
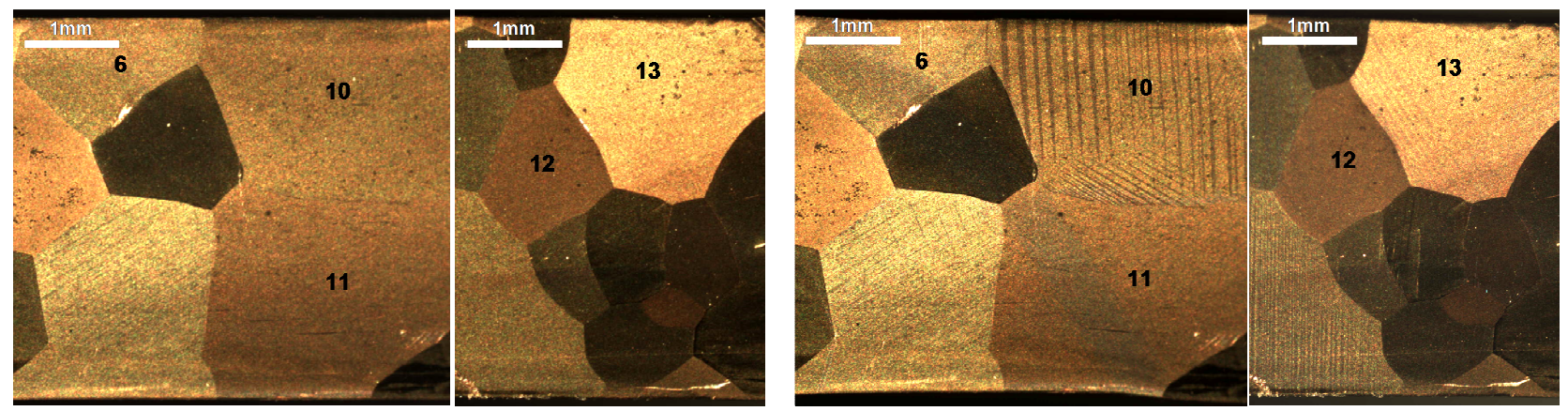

Fig. 4: Microstructure showing the formation of martensite from the initial state to loading 3, in several grains (widespread transformation in grain 10, the left and the center part fully transformed in grains 6 and 11, respectively, few plates on the left side of grain 13 and nothing in grain 12).

\section{Stress heterogeneities between crystallites}

Example experimental patterns used for the stresses analysis (at initial state and for loading 3) are shown in Fig. 5. Same conics are visible on both patterns, but Kossel lines are broader and more blurred for the deformed state. Moreover they moved due to lattice strains; it is all the more visible on the full circles whose diameters decreased.
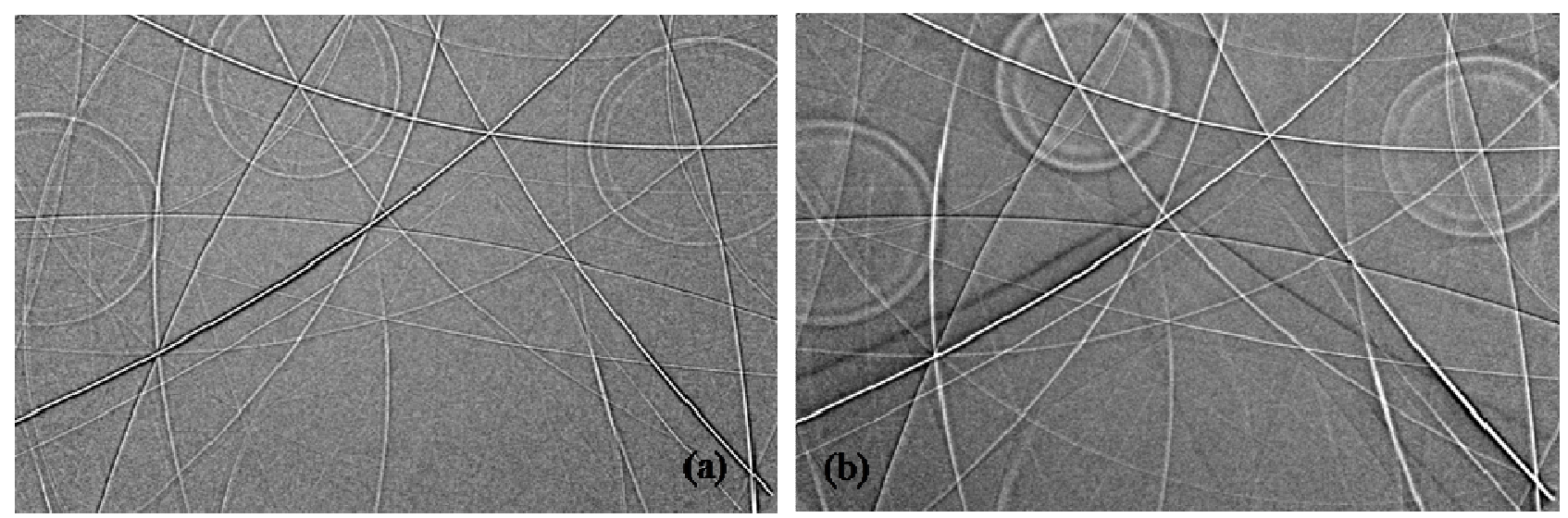

Fig. 5: Experimental Kossel patterns obtained at the initial state (a) and for loading 3 (b).

The six stress components for the 20 crystallites were calculated for three loading points plotted in Fig. 3 and after unloading, considering the initial state as reference for stress calculations. Results for the 20 crystallites are drawn in the same figure (Fig. 6). The average values for each stress component are also added. 
The average value of $\sigma_{11}\left(\sigma_{11}\right.$ along the tensile direction) increases until the loading 2 and then stabilizes. For loading 1, it is close to the applied stress (about 35MPa). For loadings 2 and 3, it is below the applied stress (about $60 \mathrm{MPa}$ compared to $80 \mathrm{MPa}$ ). The average values of $\sigma_{22}$ and $\sigma_{33}$ are close to zero for loading 1 , and decrease to about $-15 \mathrm{MPa}$ for loading 2 . At the last loading point, the average value of $\sigma_{33}$ stabilizes while $\sigma_{22}$ decreases to $-40 \mathrm{MPa}$. Malard et al. [11] already noticed (based on neutron diffraction experiments) that the average austenite stress state was lower than the macroscopic one, due to stress partitioning with martensite. It would be of interest to perform measurements in the martensite phase, but no Kossel patterns of sufficiently good quality have yet been obtained.

Considering individual stress results, large stress heterogeneities between grains are noticed, and they tend to increase with loading. The standard deviations increase from $20 \mathrm{MPa}$ for loading 1 up to about $40 \mathrm{MPa}$ for loading 3 . These trends are observed for all the stress components. The strong elastic anisotropy induces an increase of stress heterogeneities between grains, reinforced by the martensitic transformation. The maximum standard deviation for shear components is observed for $\sigma_{12}$; this is consistent with the deformation mechanism because $\sigma_{12}$ is in the loading plane.

After unloading, the average values of the six stress components are close to zero. Stresses between crystallites are about $+/-30 \mathrm{MPa}$ and $+/-15 \mathrm{MPa}$ for the normal and shear components, respectively. This is in agreement with the detected presence of residual martensite, and consistent with a non negligible macroscopic strain.
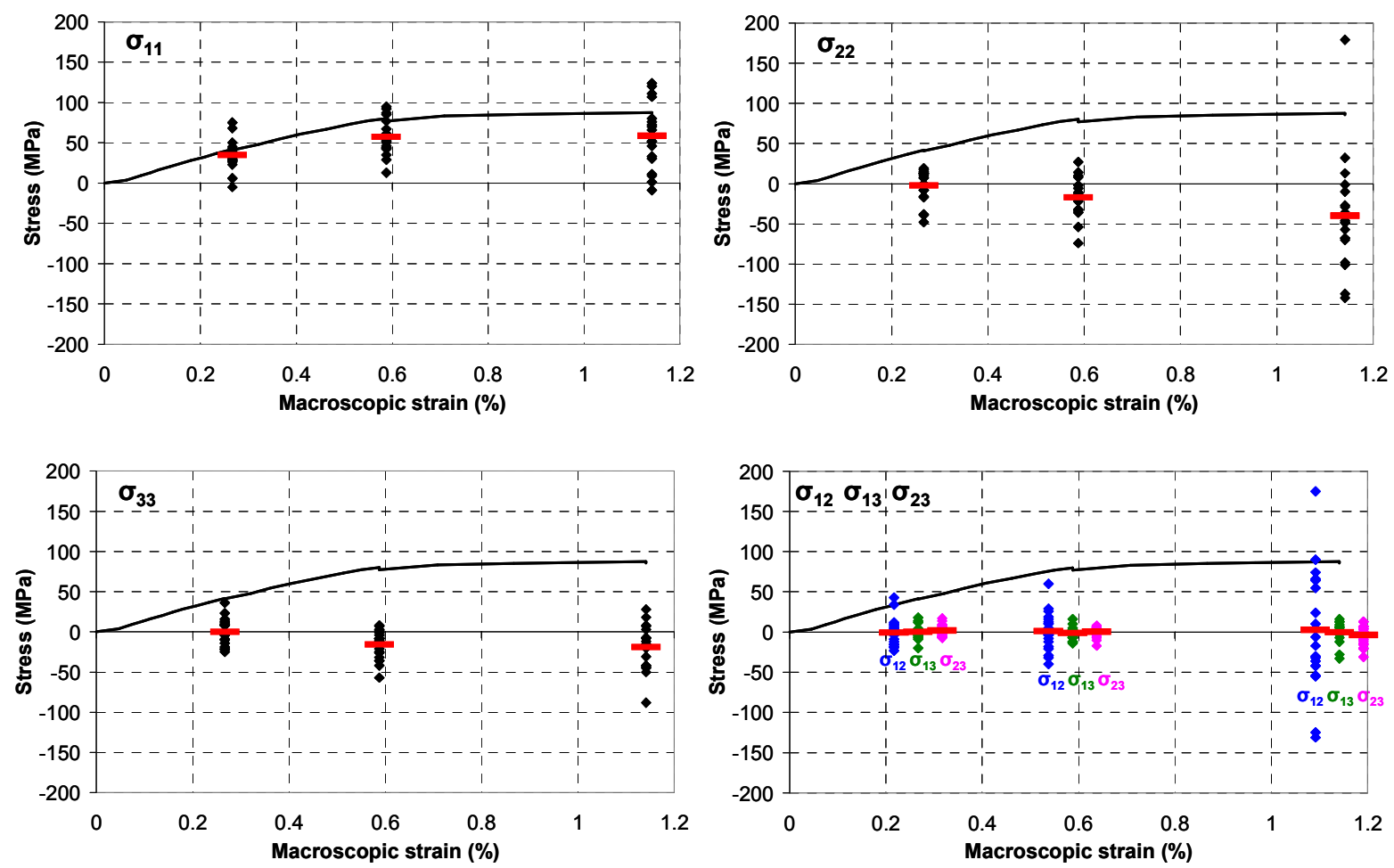

Fig. 6: Stress components (dots) determined by Kossel microdiffraction for the 20 crystallites, and the average values (dashes), relative to the applied stress.

The stress results are detailed for four particular crystallites: 6, 10, 12 and 13 (Fig. 7) which exhibit different behaviors due to different crystallographic orientations. Crystallites 6 and 10 were among the first to transform at the applied stress of $60 \mathrm{MPa}$; the crystallite 13 began to transform on its left side at $80 \mathrm{MPa}$ (loading 2). For these 3 grains, the Schmid factor for transformation is higher than 0.4. The crystallite 12 remained untransformed at the last loading point; its Schmid factor is 0.23 .

Regarding the grain 6 , its component $\sigma_{11}$ increases strongly in the elastic domain and is almost equal to the macroscopic stress. Then as martensite appears, the local $\sigma_{11}$ drops below the 
macroscopic stress and it decreases even more at the last loading point. For loading 3 , the crystallite 6 was fully transformed on its left side and not at all on its right side. The pattern was recorded near the border between these areas. The shear components are almost zero in the pure austenitic domain and start to evolve as martensite is formed; this in particular concerns $\sigma_{12}$ with its final value of about 100MPa. This phenomenon is consistent with the martensite transformation mechanism (shear of austenite). Moreover, the component $\sigma_{33}$ in the two-phase domain is not zero anymore. This observation is of importance for classical X-ray-based strain determination which uses the assumption that $\sigma_{33}$ is equal to zero.

Grains 10 and 13 have also high Schmid factors. For grain 10, two variants developed and were widespread within the whole grain. For grain 13, one variant appeared on its left side. Their stress evolution follows the same trend as that of grain 6: $\sigma_{11}$ is smaller than the macroscopic stress and it remains constant during the transformation for grain 13 while it decreases for grain 10 . In the three cases, $\sigma_{22}$ varies strongly but in different ways: positive for grain $10(150 \mathrm{MPa})$ and negative for grains 6 and 13 (between -50 and $-100 \mathrm{MPa}$ ). For the moment, we can not explain these values.

Grain 12 was oriented unfavorably. As mentioned previously, it remained untransformed during the whole loading. Unlike in the grains 6,10 and 13, its $\sigma_{11}$ is higher than the macroscopic stress whatever the loading. As the Schmid factor of this grain is low, its critical transformation stress is larger than for the other grains.
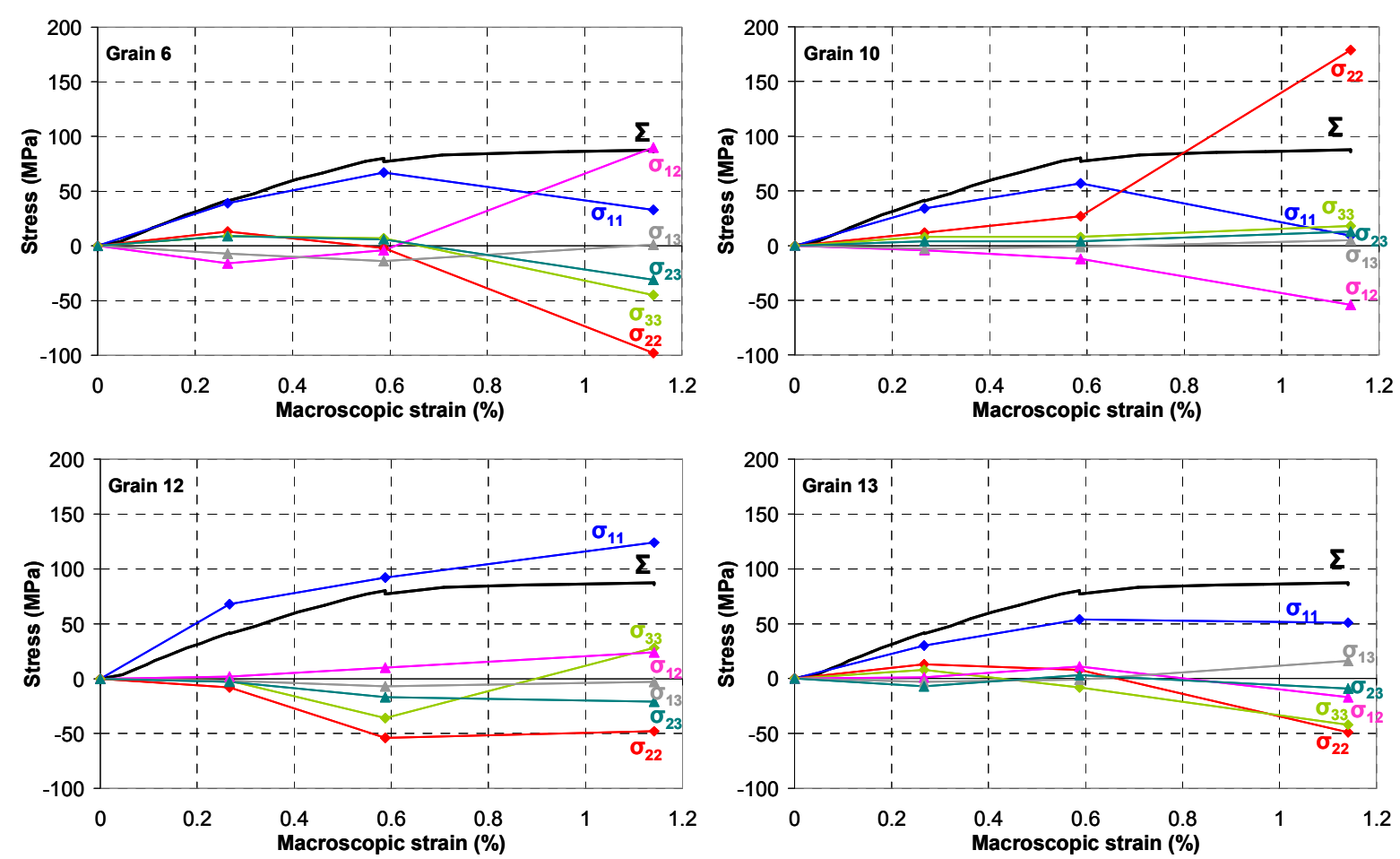

Fig. 7: Stress components determined by Kossel microdiffraction for the grains 6, 10, 12 and 13, relative to the applied stress. $\sigma_{11}$ along the tensile direction.

The results obtained in individual crystallites are in agreement with previous measurements performed by X-ray diffraction [5]. At a millimeter scale, Kaouache et al. found that high stress heterogeneities develop between grains in the elastic domain (due to elastic anisotropy of austenite) and during martensitic transformation. In that case, only two grains of a polycrystal were studied because of experimental constraints. Kossel microdiffraction allows for analyzing many more grains. Furthermore, this study indicates that, even at a local state, the assumption that the stress in the direction normal to the free surface equals zero $\left(\sigma_{33}=0\right)$ is unjustified when some fine variants of martensite co-exist with austenite. As only one measurement per grain was performed, further works are in progress to get stress heterogeneities within an individual crystallite. 


\section{Conclusion}

A Kossel microdiffraction setup, assembled in a scanning electron microscope, was used for local stress analysis in a shape memory alloy. Kossel patterns were recorded for 20 crystallites during the stress-induced martensitic transformation, and the stress components were computed. Large stress heterogeneities were obtained between grains and were compared to the crystallographic orientation and the microstructure evolution. Within individual crystallites, the martensite formation induced a stress relaxation of austenite in the tensile direction and the emergence of shear stresses as martensite variants were created. The results show that Kossel microdiffraction technique is suitable for obtaining stresses in polycrystalline samples, and therefore can be used in combination with micromechanical modeling.

As the measurements were local, results cannot be considered as average values within crystallites. The micrometric spatial resolution of the technique should be used to perform measurements within a crystallite and conclude on the intra-granular stress heterogeneities.

\section{Acknowledgments}

Work of AM was supported by National Science Center based on decision DEC2012/06/M/ST8/00449.

\section{References}

[1] H. Horikawa, S. Ichinose, K. Morii, S. Miyazaki, K. Otsuka, Orientation dependence of $\beta_{1} \rightarrow$ $\beta_{1}{ }^{\prime}$ stress-induced martensitic transformation in a Cu-AI-Ni alloy, Metall. Trans. A 19 (1988) 915923.

[2] K. Bhattacharya, R.V. Kohn, Symmetry, texture and the recoverable strain of shape-memory polycrystals, Acta Mater. 44 (1996) 529-542.

[3] E. Patoor, A. Eberhardt, M. Berveiller, Micromechanical modelling of superelasticity in shape memory alloys, J. Phys. IV 6 (1996) 277-292.

[4] S. Berveiller, B. Malard, J. Wright, E. Patoor, G. Geandier, In situ synchrotron analysis of lattice rotations in individual grains during stress-induced martensitic transformations in a polycrystalline CuAlBe shape memory alloy, Acta Mater. 59 (2011) 3636-3645.

[5] B. Kaouache, K. Inal, S. Berveiller, A. Eberhardt, E. Patoor, Martensitic transformation criteria in Cu-Al-Be shape memory alloy - In situ analysis, Mater. Sci. Eng. A 438-440 (2006) 773-778.

[6] P. Sittner, P. Lukas, D. Neov, M.R. Daymond, V. Novak, G.M. Swallowe, Stress-induced martensitic transformation in $\mathrm{Cu}-\mathrm{Al}-\mathrm{Zn}-\mathrm{Mn}$ polycrystal investigated by two in-situ neutron diffraction techniques, Mat. Sci. Eng. A324 (2002) 225-234.

[7] B. Cunningham, K.H.G Ashbee, An in-situ SEM Kossel X-ray diffraction study of pseudoelasticity, Acta Metall. Mater. 38 (1990) 2561-2565.

[8] A. Morawiec, R. Pesci, J.S. Lecomte, Semiautomatic determination of orientations and elastic strain from Kossel microdiffraction, Ceram. Trans. 201 (2008) 163-169.

[9] A. Morawiec, An algorithm for refinement of lattice parameters using CBED patterns, Ultramicroscopy 107 (2007) 390-395.

[10] S. Belkahla, Elaboration et caractérisation de nouveaux alliages à mémoire de forme basse température type Cu-Al-Be, PhD Thesis, INSA Lyon, 1990.

[11] B. Malard, P. Sittner, S. Berveiller, E. Patoor, Advances in martensitic transformations in Cubased shape memory alloys achieved by in situ neutron and synchrotron X-ray diffraction methods, C. R. Phys. 13 (2012) 280-292. 